\title{
Can probiotics prevent acute radiation-induced diarrhoea among cervical cancer patients?: the result from a randomized double-blinded placebo- controlled study
}

\author{
Ye Htut Linn, K Khine Thu, Nang Hla Hla Win
}

Department of Pharmacology, University of Medicine 1, Yangon, Myanmar

Background: Radiotherapy is the mainstay treatment of cervical cancer and the most common acute side effect is radiation-induced diarrhea (RID), which can affect up to $80 \%$ of patients. This study was aimed to investigate the effect of probiotics containing live Lactobacillus acidophilus LA-5 plus Bifidobacterium animalis subsp. lactis BB-12 for the prevention of acute RID among cervical cancer patients.

Methods: Patients who will undergo external beam pelvic radiotherapy with or without concurrent chemotherapy were randomized into probiotic or placebo group and was double-blinded. The probiotic group received the capsules each contains 1.75 billion lyophilized live bacteria which to be taken one capsule three times daily beginning from the first day to the end of radiotherapy and the placebo group received the identically appearing capsules containing starch with the same schedule. Every patient was given the standard dietary recommendations. The patients were assessed daily during radiotherapy and follow up weekly for 3 weeks after radiotherapy. Continuous variables were compared using the independent samples $t$ test and categorical variables with the Chi-square test. The $p$-values reported were two-tailed and $p$ $<0.05$ was regarded as significance. The time-to-event variables were compared with Log Rank test and described with Kaplan-Meier curves.

Results: Total 54 patients were analyzed. The incidence of diarrhoea was reduced in probiotic group than placebo group $(53.8 \%$ and $82.1 \%, \mathrm{p}<0.05)$. The mild-to-moderate and severe diarrhoea were significantly reduced in probiotic group $(\mathrm{p}<0.05)$. The use of loperamide as an anti-diarrhoeal medication was significantly reduced in probiotic group than placebo group $(\mathrm{p}<0.01)$. The difference in grade 2 abdominal pain and episodes of abdominal pain in days were also significant $(\mathrm{p}<0.001)$. But the onset of diarrhoea, interruption of radiotherapy due to diarrhoea, dose of loperamide and its onset and patients' conditions during the 3 weeks follow ups were not significantly different between the two groups.

Conclusion: Supplementation of probiotics containing live Lactobacillus acidophilus LA-5 plus Bifidobacterium animalis subsp. lactis BB-12 is an easy and effective way to reduce the incidence and severity of RID in cervical cancer patients. 